\title{
THERMAL DRILLING OPTIMIZATION IN MULTI HOLE PLATES
}

\author{
Jan Kosmol \\ Silesian University of Technology, Faculty of Mechanical Engineering, Department of Machine Technology \\ Konarskiego 18A, 44-100 Gliwice, Poland \\ Corresponding author: Jan Kosmol, jkosmol@polsl.pl
}

\begin{abstract}
The article presents the results of simulation of thermal deformations by the finite element method for round multi-hole plates used in heat exchangers. The heat generated when drilling holes causes thermal deformation of these objects, which contributes to errors in the location of the holes. Obtained results of simulation were compared for different drilling strategies (the studies considered 24 different strategies). It was found that the maximum drilling temperatures according to different strategies may differ by up to $100 \%$. Similar conclusions can be drawn for thermal deformations. The general conclusion that results from the conducted research indicates the need to choose a strategy that ensures the symmetry of the drilled holes in relation to the axis of symmetry of the object. Then, both thermal deformation and maximum temperature are the smallest. The thus identified thermal deformations can
\end{abstract}

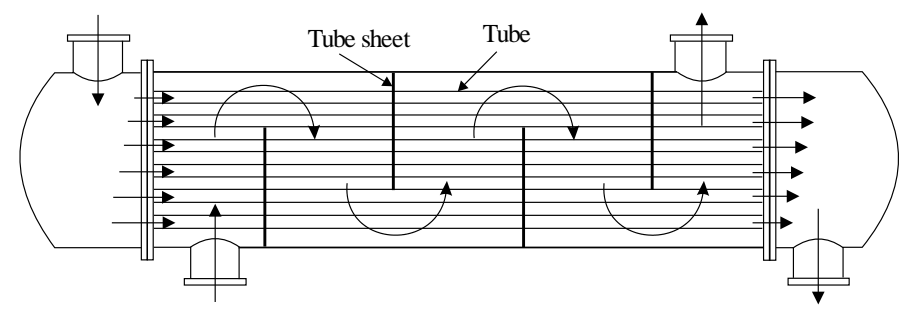

a) form the basis for the correction of the coordinates of the holes on a CNC multi-spindle drilling machine.

Key words: thermal deformation, multi-hole objects, optimization, FEM

\section{INTRODUCTION}

In industry, especially in the energy sector, it is not uncommon to find large-size objects, the components of which are made in machining technology. One of them are heat exchangers (Pudlik, 2012) in which heat is transferred from a fluid with a higher temperature to a fluid with a lower temperature. Figure 1 shows an example of such an exchanger, called a recuperator.

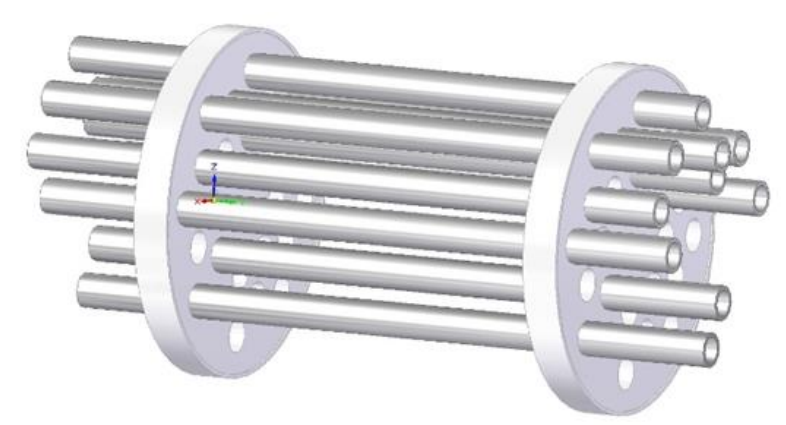

b)

Fig. 1. Diagram of a heat exchanger, the so-called recuperator (a), arrangement of tubes (b)

The recuperator contains hundreds or even thousands of tubes of small diameters $(10-15 \mathrm{~mm})$ through which the fluid flows at a higher temperature. These tubes flow around the cooler fluid which is heated by heat transfer. Due to the length of the exchanger (a few meters) and due to the relatively low stiffness of the pipes (a pipe with a length of several meters and a diameter of several millimeters), it deforms significantly under its own weight and fluid weight. Therefore, the exchanger has several partitions (plates with many holes and the so-called tube sheets, which support all the tubes and keep them in the appropriate spatial configuration. The partitions, depending on the size of the exchanger, are round, thin-walled plates with diameters from $1 \mathrm{~m}$ to several meters. The holes in these partitions are made by machining, by drilling. Due to the technological requirements, holes must be made and deepened on both sides of the board. Figure 2 shows an example of a partition with several hundred holes. Typical partitions have the following geometric features:

- all holes are of the same diameter, but these holes must contain a small chamfer on both sides of the plate (Figure 2(b)),

- all holes are spaced according to the same scale,

- the boards have a thickness of up to several dozen millimeters, which qualifies them as slender objects. 


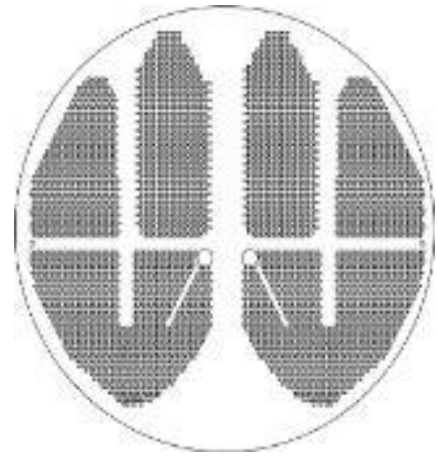

a)

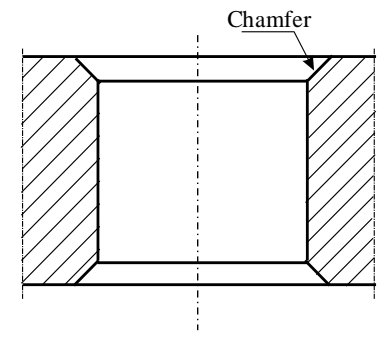

b)
Fig. 2. An example of a heat exchanger partition (a) and a cross-section of a single hole (b)

The production of such plates, and especially the drilling of several thousand holes, presents at least two economic and technological problems. The economic problem is related to the production time of all holes. The production time is from several to several hours, which indicates the advisability of its optimization. The issue of optimization of cutting processes is presented, among others, in (Wittbrod, 2013), (Pająk and Wieczorowski, 1982), while the problem of optimizing drilling a large number of holes was presented by the author in (Kosmol, 2021). Typically, holes are made on numerically controlled multi-spindle drilling machines (Collective work, 1974).

Figure 3 shows schematically a five-spindle CNCcontrolled drilling machine. It enables individual control of the position of each headstock in the $\mathrm{X}$ axis. In this way, it is possible to simultaneously drill 5 holes freely spaced apart. The optimization problem is presented as follows (Kosmol, 2021): if there are $\mathrm{Nj}$ holes in the $\mathrm{j}$-th row of holes, how to divide this number among all spindles to make the time of making all holes $\mathrm{Nj}$ as short as possible?

In the proposed optimization algorithm, a slightly different optimization criterion was used, namely the degree of utilization of available spindles (DUAS). In the special case, when the number of holes in the $\mathrm{j}$-th row $\mathrm{Nj}$ is divisible by the number of spindles, the degree of use of the spindles is $100 \%$ and then the time of making all the holes is the shortest. In any other case, the DUAS will be lower than $100 \%$ and then an optimal solution should be sought.

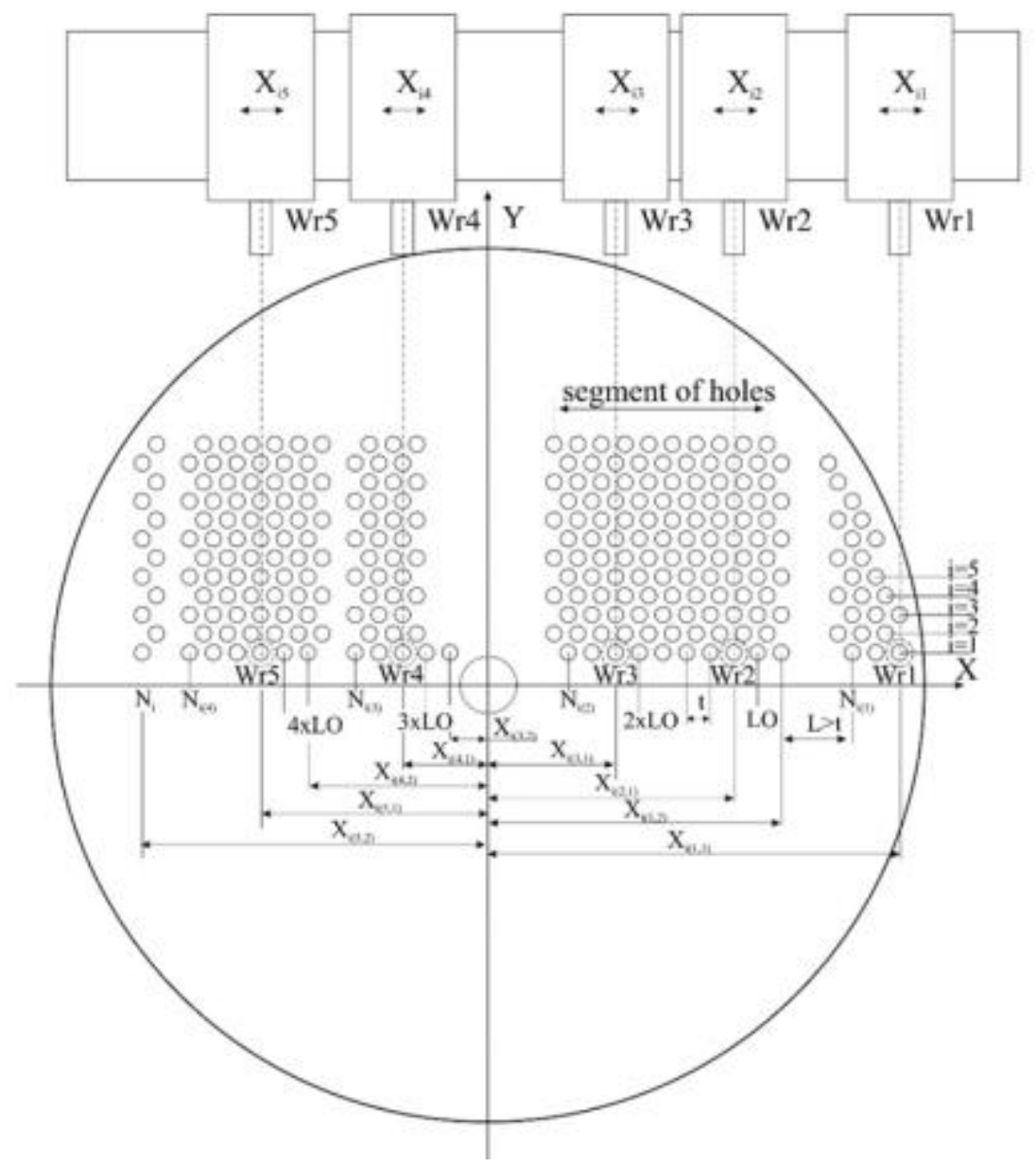

Fig. 3. Example of a typical energy partition with multiple holes: $\mathrm{Wr}_{1}, \mathrm{Wr}_{2}, \mathrm{Wr}_{3}, \mathrm{Wr}_{4}, \mathrm{Wr}_{5}-\mathrm{symbols}_{\text {of }}$ spindles, $\mathrm{N}_{\mathrm{i}}, \mathrm{N}_{\mathrm{i}(1,1)}$, $\mathrm{N}_{\mathrm{i}(3,1)}, \mathrm{N}_{\mathrm{i}(4,1)}, \mathrm{N}_{\mathrm{i}(5,1)}$ - number of holes, LO - number of holes manufactures by one spindle (Kosmol, 2021) 
In (Kosmol, 2021) an optimization algorithm was proposed that allows for the optimal division of all holes in the $\mathrm{j}$-th row into individual spindles. The end result of such optimization is the graph in
Figure 4 (Kosmol, 2021), which shows the dependence of the degree of use of DUAS spindles and the optimal number of holes per single LOopt spindle on the number of holes $\mathrm{Nj}$ in the $\mathrm{j}$-th row.

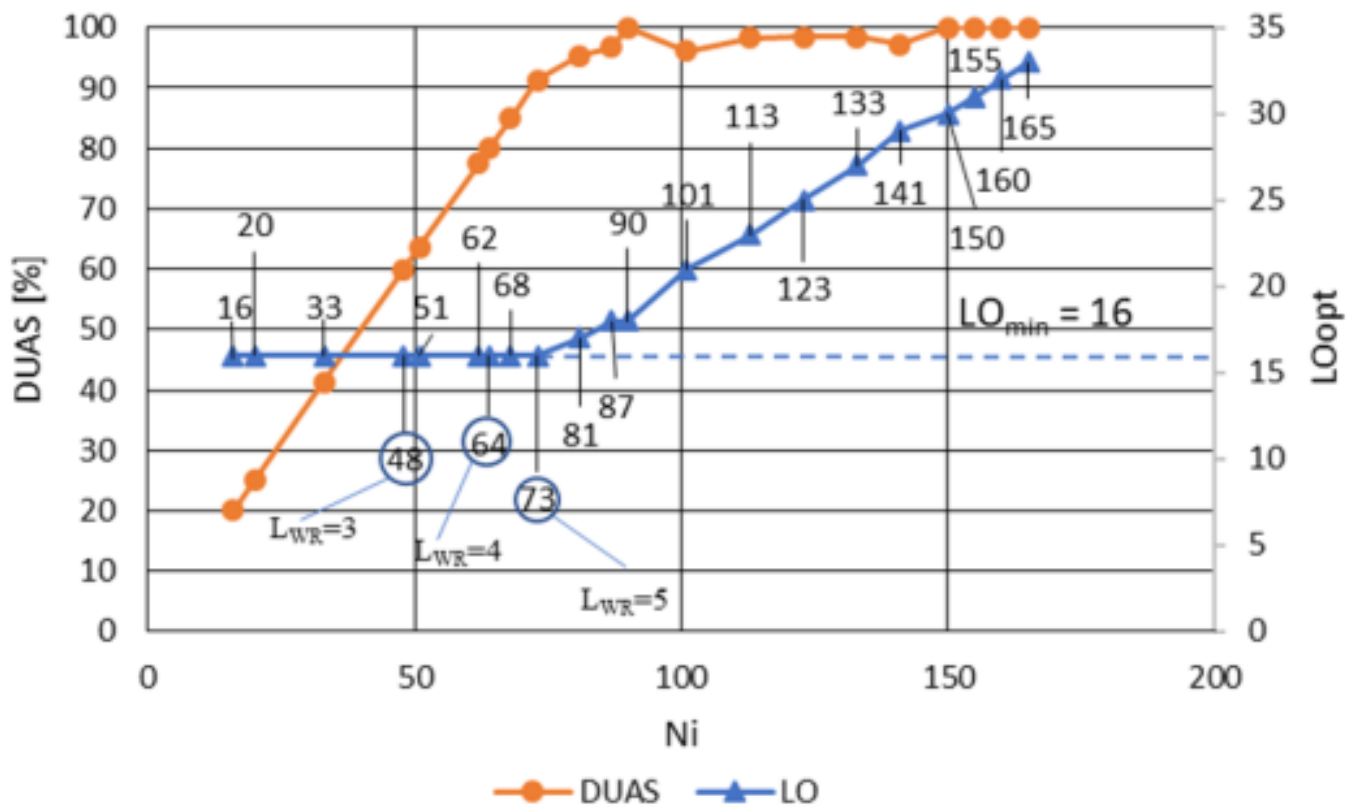

Fig. 4. Influence of the number of holes $\mathrm{N}_{\mathrm{i}}$ in a single partition row on the optimum number of $\mathrm{LO}_{\text {opt }}$ holes and the utilisation rate of DUAS spindles; $\mathrm{L}_{\mathrm{WR}}$ - number of active spindles, $\mathrm{LO}_{\min }$ - minimum number of holes per spindle

(Kosmol, 2021)

Making such a large number of holes by drilling them in a relatively flaccid object entails another, significant problem, namely the technological one. This problem is due to the fact that cutting heat is released when the holes are drilled, which contributes to thermal deformation of the entire plate. Illustratively, it is shown in Figure 5.

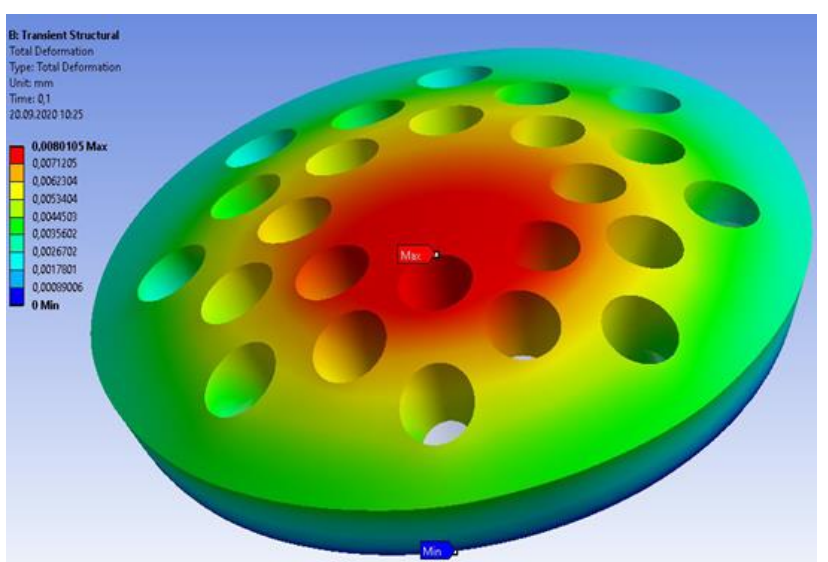

Fig. 5. Image of a thermally deformed test plate as a result of simulation with the Finite Element Method of drilling 24 holes

Characteristic for the image of the heat-deformed plate is the spatial location of the axes of individual openings, which are no longer parallel to the plate axis. As a result, the distances between the axes of the holes differ from what is expected and the axes of the holes are deviated from the axis of the plate by certain values of angles.

Since the plate, after making the holes, on one side must be turned in such a way that it can be drilled on the other side in order to make chamfers (Figure 2(b)), the chance that numerically controlled individual axes of the headstocks will hit the axes of the holes are small. As a result, the chamfers will be made asymmetrically with respect to the axis of the holes. One of the possible ways to minimize the adverse effects of thermal deformation of the plate is to predict the position of the axis of the holes by numerical simulation. If, by means of numerical simulation, it is possible to identify the position of the axes of the holes, then in the numerically controlled machine tool it will be possible to use the function of correcting the position of the headstock axes, according to the simulation results, and thus reduce the adverse effects of thermal deformations. Since the size of thermal deformation of the plate significantly depends on the order of drilling of individual holes, an optimization problem can be formulated in the form of a question: what sequence of hole drilling leads to the smallest thermal deformation of the plate? 


\section{HOLE DRILLING STRATEGIES}

Analytical search for the most favorable solution seems to be difficult or even impossible. Each of the drilled holes is a heat source, with the individual heat sources becoming active at different times. Therefore, it was assumed that the numerical method in the form of the Finite Element Method will be used. It enables modeling of the heat flow for each of the openings and relatively easy definition of the moment in time when a given heat source becomes active. What is its disadvantage is the duration of the simulation. The conducted research is exploratory. The researchers looked for an answer to the question to what extent the different drilling strategies affect the thermal deformation of the plate? For this purpose, a test plate with 16 holes was defined (Figure 6).

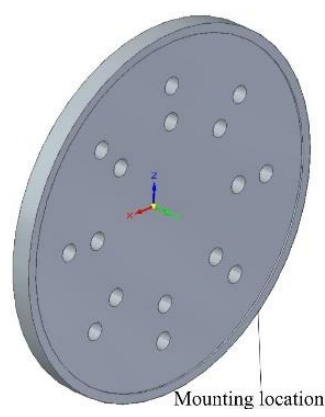

a)

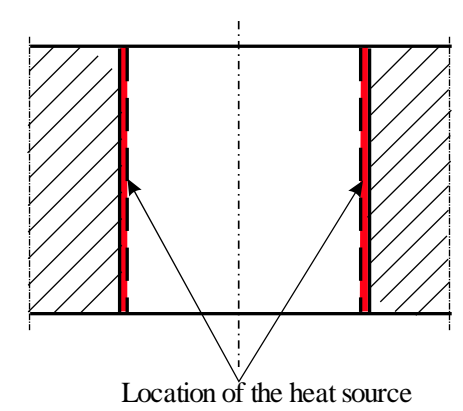

b)
The number of holes is limited to 16 due to the simulation time. A single simulation on $\mathrm{PC}$ hardware, depending on the drilling strategy of individual holes, lasted up to 50 hours.

This significantly limited the ability to perform more drilling strategies. In order to avoid the influence of the method of fixing the plate on the machine tool, it was assumed that the plate model was deprived of degrees of freedom on the surface of a narrow ring on the outer diameter of the plate.

The following drilling strategies were considered (Figure 7):

- the strategies S1 and S4 relate to drilling single holes in two different sequences.

- the strategies S2 and S5 concern simultaneous drilling of two holes in the same order as in the strategies $\mathrm{S} 1$ and $\mathrm{S} 4$.

- the strategies S3 and S6 concern the simultaneous drilling of four holes in a similar sequence as in the strategies S1 and S3.

Fig. 6. Diagram of the 16-hole test plate (a) and the location of the heat source in the hole (b)
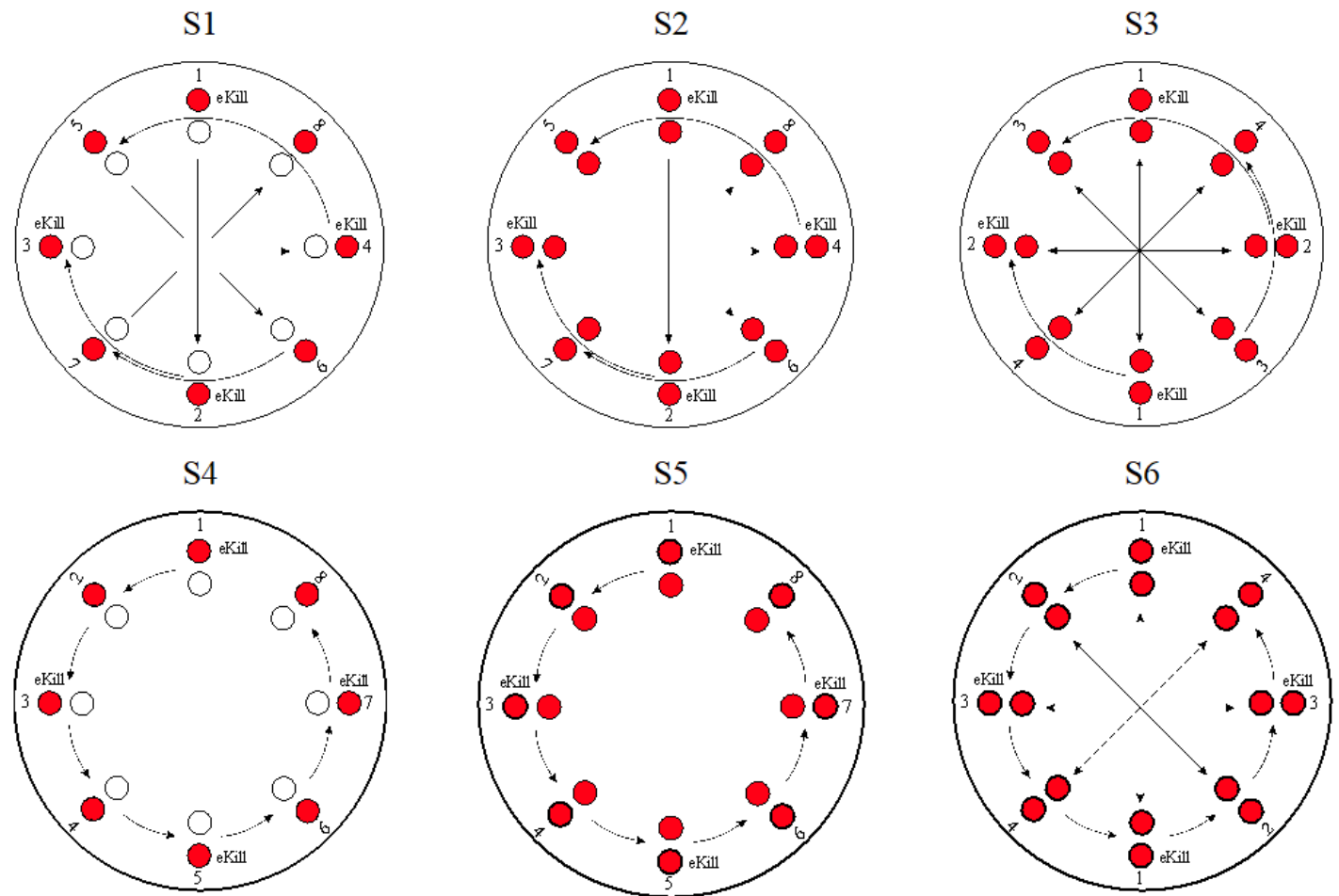

Fig. 7. Strategies for drilling holes in a 16-hole plate; eKill-marking holes modeled in the disappearing material mode 
Such a selection of a strategy enables the assessment of both the number of simultaneously drilled holes and the sequence of their drilling.

The Finite Element Method simulation studies were carried out for two substantially different hole drilling modeling methods, namely:

a) In the simplified version, when the chip formation during drilling was not modeled, and only on the internal surfaces of the holes, heat sources were placed, the power of which was equal to the cutting power. This method of FEM modeling significantly shortens the simulation time, although it does not fully correspond to the drilling phenomenon (the plate is mechanically less stiff and all the heat flows to the plate and the environment).

b) In the extended version, when the phenomenon of the disappearance of the drilled material was modeled, i.e. the amount of material in the hole decreased with time (in Figure 7, the holes in which the disappearance of the material was modeled were marked as eKill). This modeling method substantially changes the heat distribution as some heat escapes with the disappearing drill material. Moreover, the maximum temperature, which was one of the hole drilling indicators, was concentrated in the disappearing material and not in the area of the plate (this was the case in the simplified version).

Simulations with modeling of the loss of drilled material concerned only 4 to 8 out of 16 holes. The remaining openings were modeled in a simplified version. The reason for this was the duration of a single simulation. As already mentioned, for the four extended modeled holes, it was 50 hours or more. If we wanted to model all 16 holes in the extended mode, the time of a single simulation would exceed 200 hours.

\section{MODELING OF HEAT FLOW AND THERMAL DEFORMATION BY THE FINITE ELEMENT METHOD}

Simulation tests using the Finite Element Method were carried out in the Ansys 2020R2 system (Ansys, 2019), because it allows, inter alia, material decay modeling (eKill function) (https://ansyshelp.ansys.com). Coupled thermomechanical analysis was used, it means combinations of Transient Thermal analysis (heat transfer) and Transient Structural analysis (thermal deformation), (Porada, 2020), Figure 8. Heat transfer analysis (Transient Thermal) requires the definition and allocation of heat sources. For this purpose, cutting power while drilling a single hole (Olszak, 2008) was calculated and assigned to the Heat Flow heat source. The heat sources themselves were placed on the inner walls of each opening (Figure 6(b)).

Each heat source was assigned a time point of its activation and the time of its activity. This is illustrated in Figure 9 for an example S4 strategy. For the assumed drilling conditions (Olszak, 2008), the activity time of a single heat source was $2 \mathrm{~s}$.

After completing the heat flow analysis (Transient Thermal), the Imported Load function was used, or actually one of its activities: Imported Body Temperature, which enables automatic transfer of temperature distribution from Transient Thermal analysis to Transient Structural analysis. From that moment, the analysis of thermal deformation proceeded according to the temperature distribution obtained from the Transient Thermal analysis.

The classic Ansys FEM modeling method is based on the Ansys Workbench graphical interface. On the other hand, the Ansys system also has its own programming language APDL (Ansys Parametric Design Language), which enables the formulation of Finite Element Modeling tasks in text form, the so-called Commands. Starting from Ansys 2018, the eKill command is available, which allows you to remove a defined part of the material from the modeling area. In this study, we used this command to remove material from a drilled hole. As already mentioned, the eKill command was used only for four to eight holes, marked in Figure 7 eKill, because the simulation time with such a command increases disproportionately. For example, on Figure 10 the eKill command occurs four times, i.e. from 0 to 16 seconds of the simulation, while from 16 seconds to the end of the simulation, the eKill command no longer occurs.

The example in Figure 10 shows that the eKill command affects both the temperature (the temperature is higher than without the eKill command) and the waveform itself (discontinuities in the waveforms are observed). It was assumed in the research that heat flow occurs only as a result of convection. Other methods of heat transfer, e.g. by radiation, have been omitted.

Should be noted that cutting forces generated during drilling change the state of deformation of the plate. Modeling with FE did not take into account these forces. The eKill method was used to model disappearing of materials but not the cutting force. 


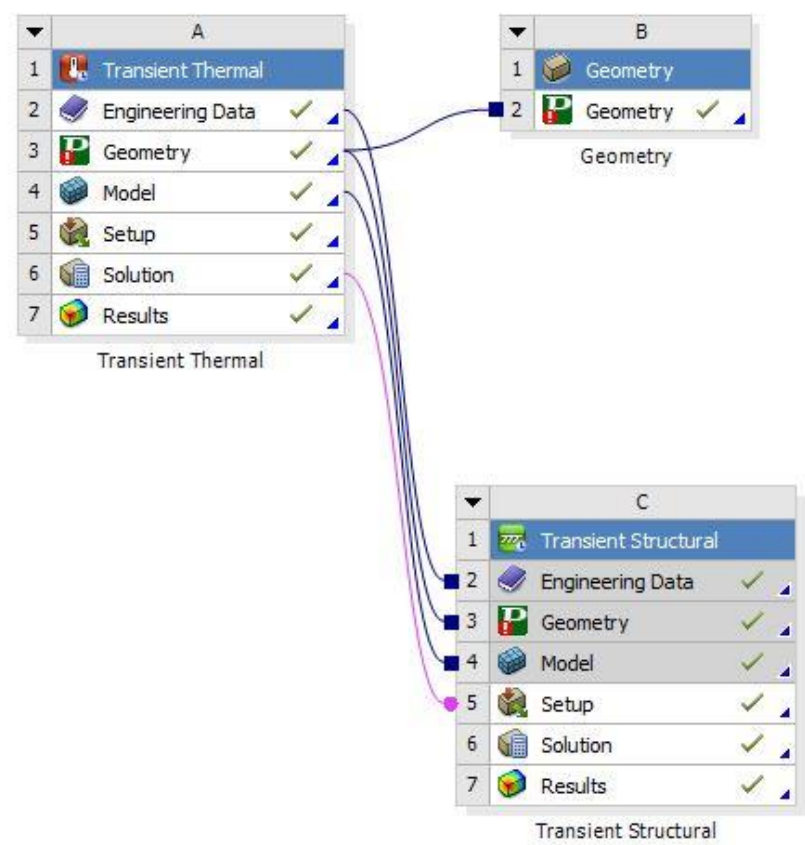

a)

Fig. 8. Combined Transient Thermal and Transient Structural analyzes in Ansysy 2020R2

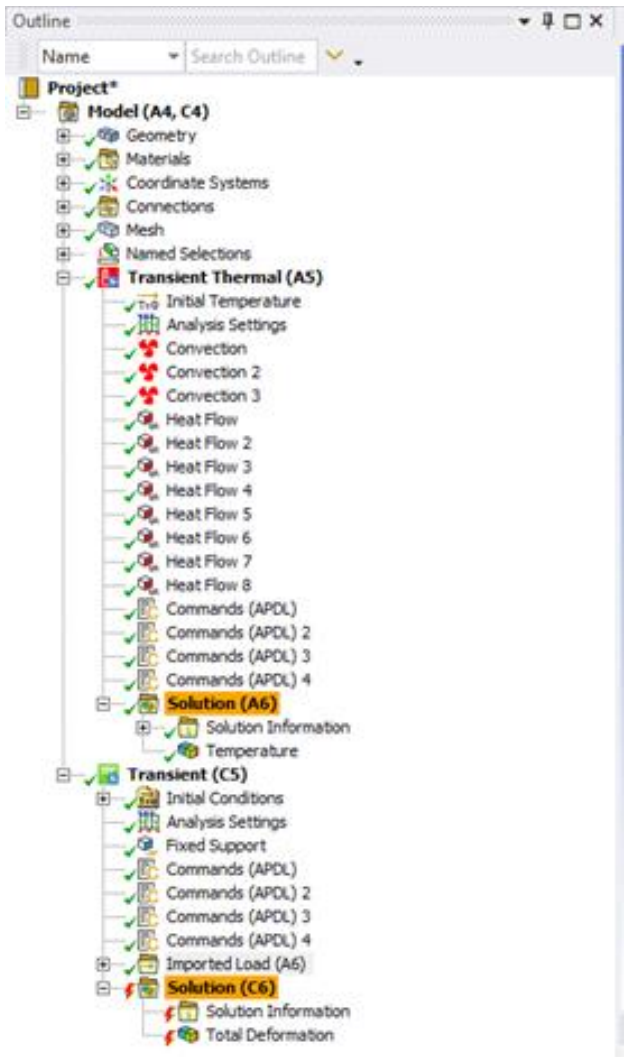

b)

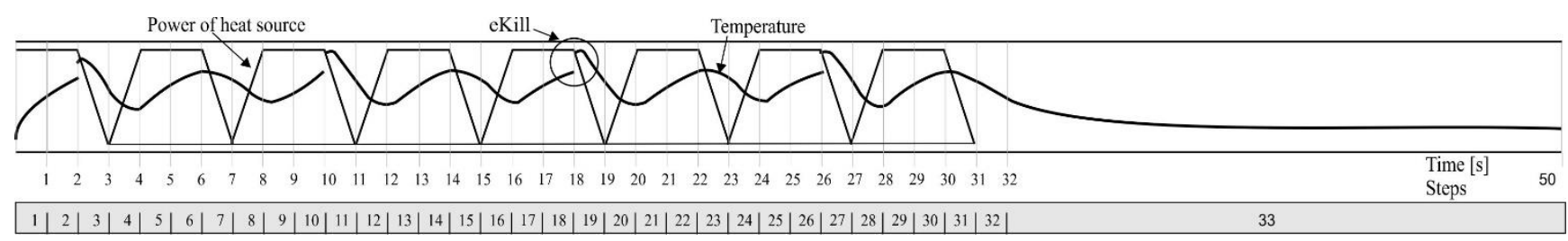

Fig. 9. An exemplary heat source power curve for the S4 strategy

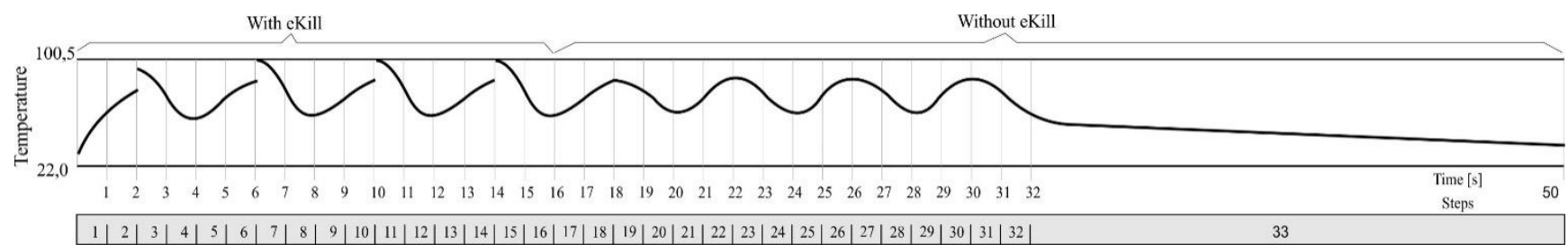

Fig. 10. An example of the temperature course with the use of the eKill command (from 0 to 16 seconds of the simulation) and without the eKill command (from 16 seconds to the end of the simulation)

\section{RESULTS OF FEM SIMULATION TESTS}

The purpose of the simulation studies was to determine whether and to what extent the change of the drilling strategy affects the temperature distribution of the multi-hole plate and its thermal deformation. Therefore, the following criteria values were adopted for the quantitative assessment of such an impact, namely:

- maximum plate temperature,

- average plate temperature,

- plate thermal deformation after 50 seconds of simulation. It means that after 30 seconds 8 holes will be made and after last 20 seconds the plate reached the set temperature,

- time of making all holes.

The simulations were carried out for the strategies presented in Figure 7, both with the presence of the eKill command (6 simulations) and without the presence of the eKill command (6 simulations). Moreover, each of the 12 simulations was repeated for two different sequences (compare simulations S1 with S4, S2 with S5 and S3 with S6 in Figure 7). As a result, 24 test results were obtained, on the basis of which conclusions were drawn regarding the influence of the strategy on the temperature and 
thermal deformation of the plate. Figure 11 shows the courses of temperatures and thermal deformation in total for four examples, for

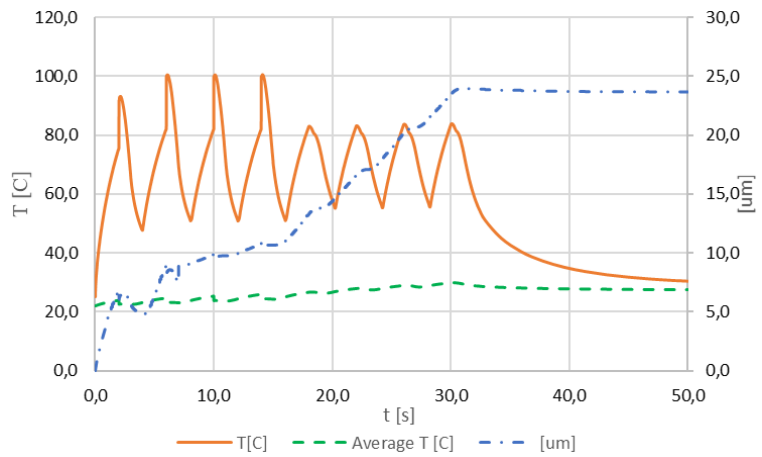

a)

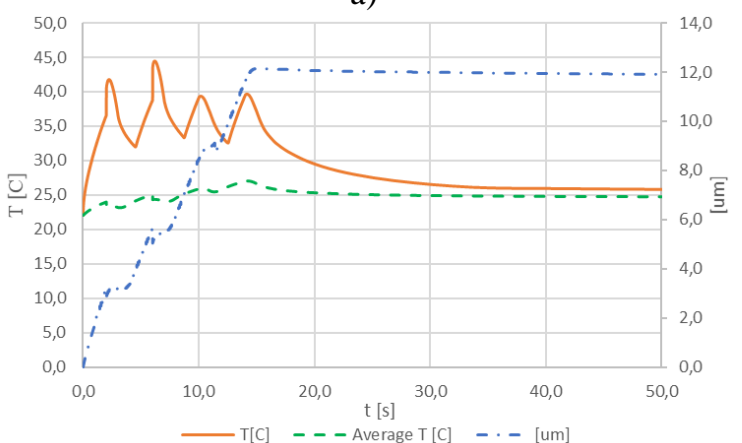

c) the S1 and S4 strategies and for the S3 and S6 strategies with the eKill command application.

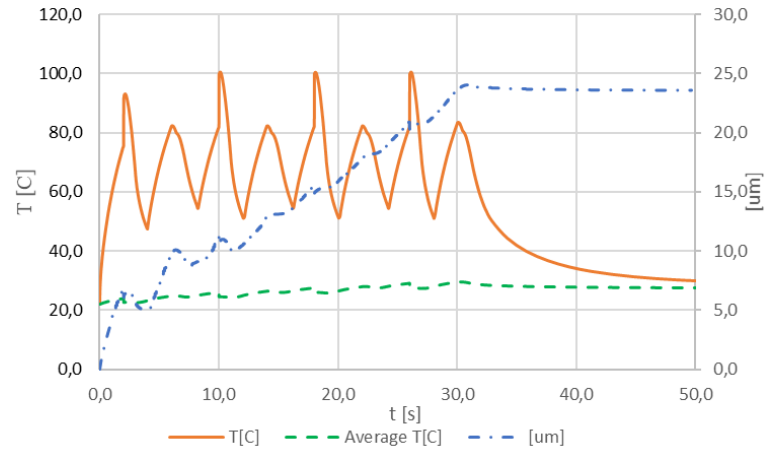

b)

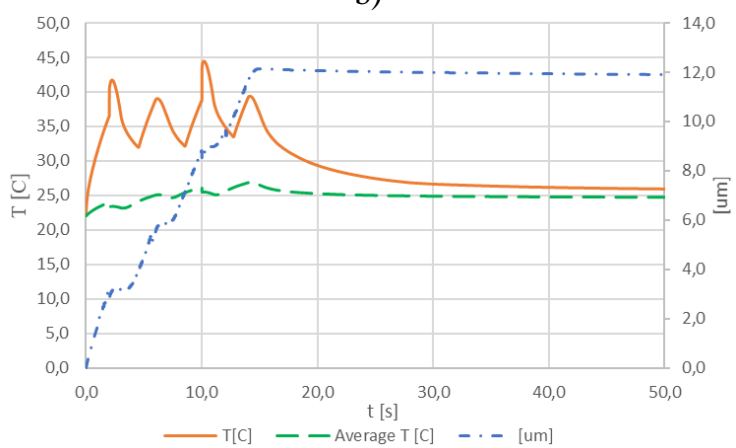

d)

Fig. 11. Time courses of temperatures and thermal deformations with the eKill application: a) S1, b) S4,

c) S3, d) S6; T - maximum plate temperature, Average T [C] - average plate temperature, um - thermal deformation

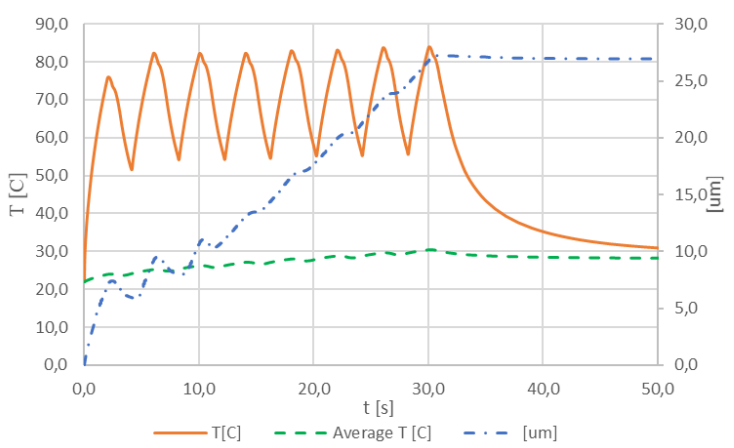

a)

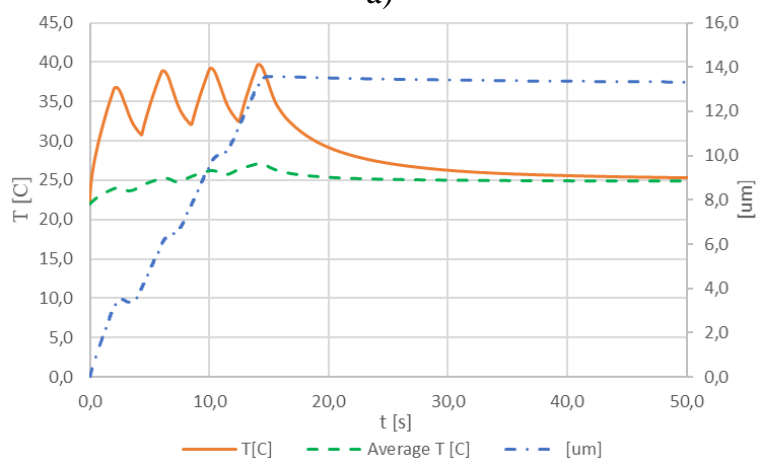

c)

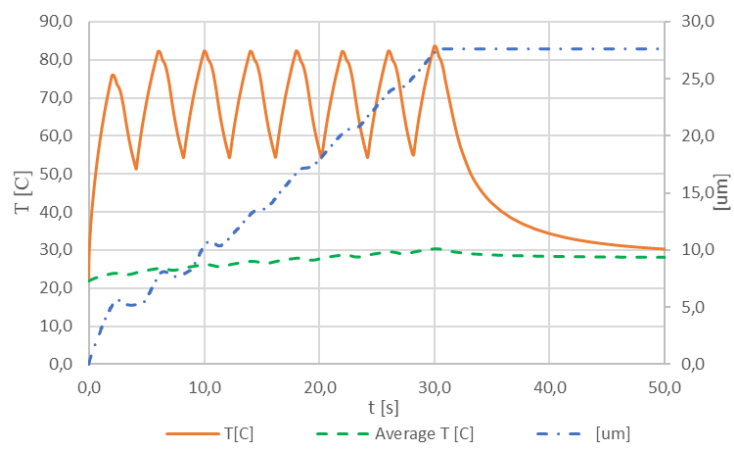

b)

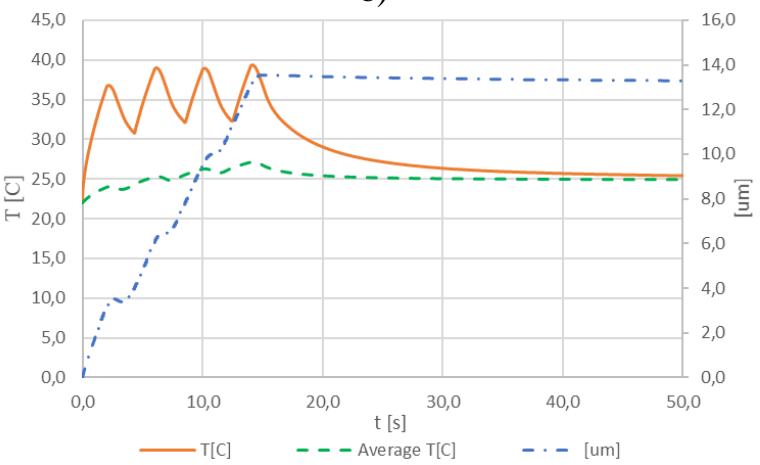

d)

Fig. 12. Time courses of temperatures and thermal deformations without the application of the eKill command: a) S1, b) S4, c) S3, d) S6; T - maximum plate temperature, Average T [C] - average plate temperature, um - thermal deformation 
On the other hand, Figure 12 shows the same temperature and thermal deformation courses, but without the eKill command.

On the basis of such simulation test results, graphic relations of maximum temperature, average temperature and thermal deformation of the plate from the strategy were developed.

Figure 13 shows these relationships for simulation studies with the eKill command application, on Figure 14 without $e$ Kill application.

The maximum plate temperature does not necessarily occur at the same point in the plate. On the contrary, for different drilling strategies, the location of the maximum temperature will vary and is not further defined.

The plate thermal deformation was identified at the time $t=50 \mathrm{~s}$. It was assumed that it corresponds to the steady state, i.e. after the plate has cooled down to the ambient temperature. In fact, the steady state followed a much longer period of cooling down, but then the simulation duration increased several times, up to several hundred hours, which was technically difficult to achieve.

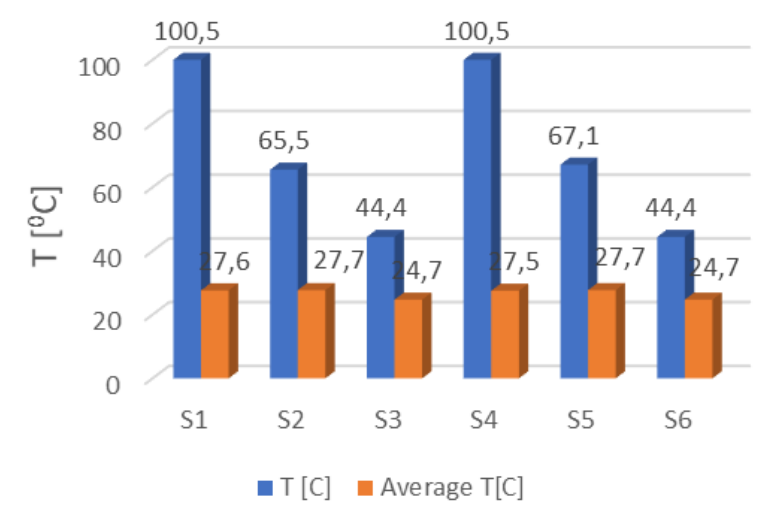

a)

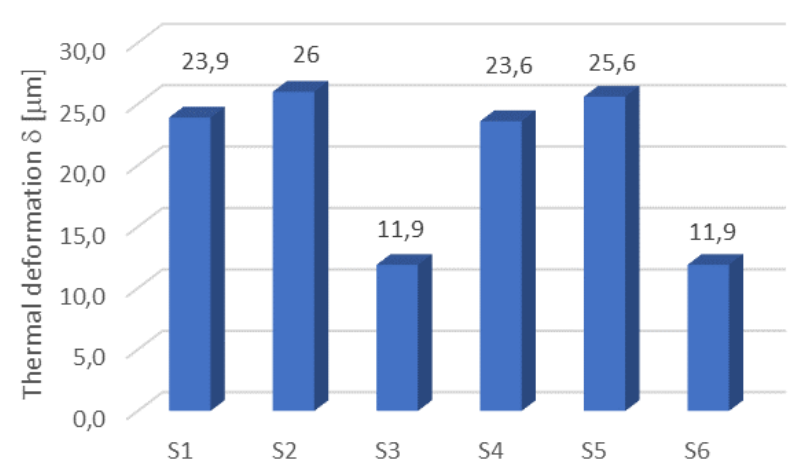

b)

Fig. 13. The impact of the drilling strategy on: maximum and average plate temperature (a) and plate thermal deformation (b) with the $e K i l l$; Average T[C] - average plate temperature, $\delta$ - steady state thermal deformation

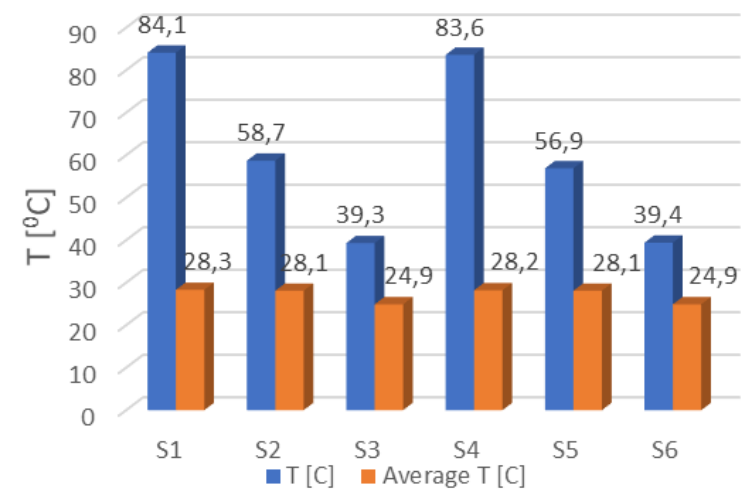

a)

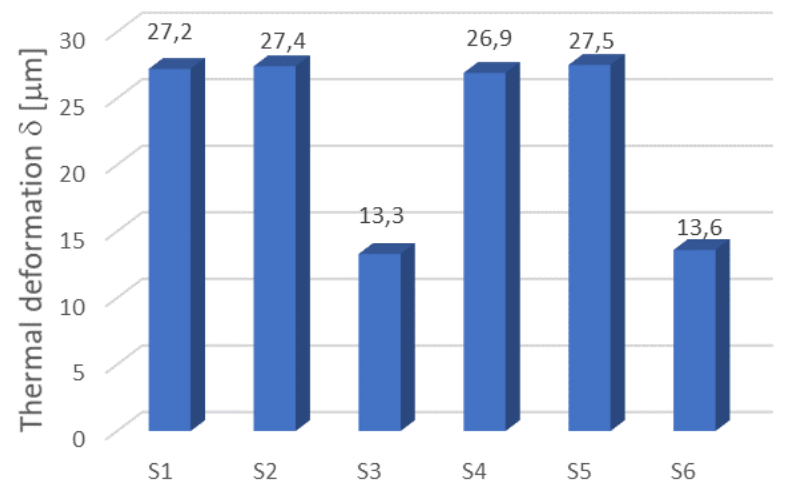

b)

Fig. 14. The impact of the drilling strategy on: maximum and average plate temperature (a) and plate thermal deformation (b) without eKill command application; Average T [C] - average plate temperature, $\delta$ - steady state thermal deformation

For a complete and correct interpretation of the test results presented in Figure 13 and Figure 14, Figure 15 shows the relationships between the time of making all the holes for each strategy (it was assumed that the execution of a single hole takes $2 \mathrm{~s}$ and that the time between making the next hole is $0 \mathrm{~s}$ ) and the time of making all holes and the maximum temperature.

The following conclusions can be drawn from Figure 15:
- The time of making all (in this example 16) holes significantly depends on the adopted strategy. Generally, the more holes are drilled simultaneously, the faster the time it takes to make all the holes. This is an obvious conclusion.

- On the other hand, the conclusion resulting from Figure 14(b) is valid, however obvious. The longer the drilling time, the higher the maximum temperature. This is due to the fact that more heat will penetrate into the plate over a longer period of time and thus its temperature will increase. 
- As the drilling time increases, the maximum plate temperature also increases. Between strategies $\mathrm{S} 1$ and S3 and between strategies S4 and S6, the execution

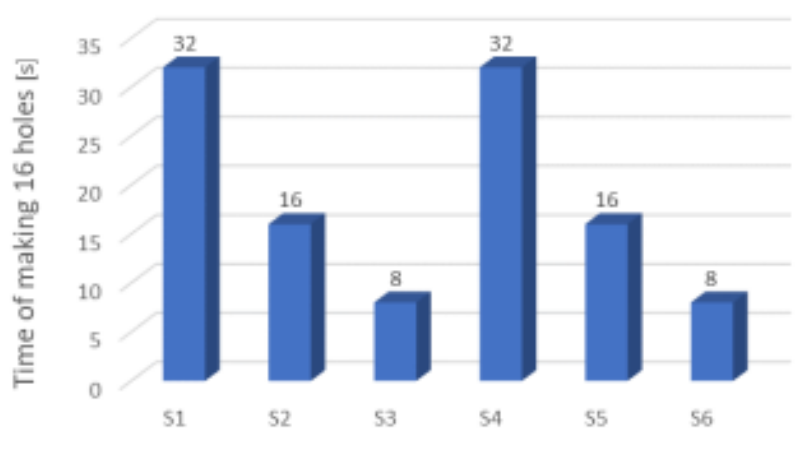

a) time for all 16 holes decreases approx. 4 times and the maximum temperature decreases by approx. 2 times.

Fig. 15. Time of making 16 holes for the adopted strategies (a), relationship between the time of making 16 holes and the temperature (b); Average T [C] - the average temperature of the plate

The analysis of the results presented in Figure 13 and Figure 14 allows for the following conclusions:

- The maximum plate temperature depends on the strategy adopted. For the research carried out, it differs by over $100 \%$, regardless of whether the eKill command was included or not.

- The greater the number of simultaneous holes, the lower the maximum temperature. For example, for an S1 strategy without eKill, only a single hole is made and the maximum temperature is $84^{\circ} \mathrm{C}$, while for the S2 strategy, when 2 holes are made simultaneously, the maximum temperature drops to approx. $57-58^{\circ} \mathrm{C}$. However, taking into account the eKill command, the temperature for the $\mathrm{S} 1$ strategy is approx. $100^{\circ} \mathrm{C}$ and for the $\mathrm{S} 2$ strategy it is approx. $65^{\circ} \mathrm{C}$.

- Referring to the average plate temperature, which has a more significant impact on thermal deformation, a conclusion similar to the conclusion from the effect of the maximum temperature can be drawn. However, the impact is less acute, i.e. no significant difference is seen between the $\mathrm{S} 1$ and $\mathrm{S} 2$ strategies and between S4 and S5. Only for the S3 and $\mathrm{S} 6$ strategies is the decrease in the average temperature of approx. $30^{\circ} \mathrm{C}$ visible.

- Comparing the maximum temperatures for simulations taking into account the eKill command with simulations that did not take this mechanism into account, it can be concluded that modeling with the eKill command generally gives higher temperatures, e.g. for the S1-eKill strategy the temperature is about $100^{\circ} \mathrm{C}$ and $84^{\circ} \mathrm{C}$ for $\mathrm{S} 1$-without eKill and for the S6 strategy, these differences are clearly smaller, namely $44.4^{\circ} \mathrm{C}$ and $44^{\circ} \mathrm{C}$. However, for average temperatures, the values for both modeling methods are similar, i.e. $27.6 / 28.3^{\circ} \mathrm{C}$ for $\mathrm{S} 1$ and $24.7 / 24.9^{\circ} \mathrm{C}$ for $\mathrm{S} 6$.
- Plate heat deformation also significantly depends on the strategy, although the effect is slightly weaker. Comparing the strategies S1 with S2 or S4 with S5, no significant differences in thermal deformation are noticed. They are at the level of $2 \%$. But if we relate these strategies to the S3 strategy or to the S6 strategy, the differences are already fundamental, as they reach $50 \%$ or more. Here again, the strong influence of the total drilling time for all holes becomes apparent, which is shorter than that of the strategies S1 and S2 or S4 and S5 by 2 to 4 times for the strategies S3 and S6. Undoubtedly, such an effect is also influenced by much lower temperatures for the $\mathrm{S} 3$ and S6 strategies compared to the S1i S2 or S4 and S5 strategies (for the maximum temperature it is approx. 50\% decrease).

- Comparing strategies S1 with S2 or S4 with S5, when the time of making all the holes is the same but the number of simultaneously drilled holes is different, we observe a slight increase in the thermal deformation of the plate (approx. 2\%) for those strategies for which the number of simultaneously drilled holes is greater. This is so even though the maximum temperature in the latter case (S2 or S5) is lower than in the first case (S1 or S4).

\section{CONCLUSIONS}

The article deals with the problem of thermal deformation of multi-hole and large-size thin-walled plates. It has been found that the cutting heat generated when drilling the holes contributes to the thermal deformation of the plate itself, which may pose problems in achieving the expected accuracy of the position of the axes of the holes. It has been found that this can be prevented to some extent by selecting 
an appropriate drilling strategy, i.e. an appropriate number of simultaneously drilled holes and the appropriate sequence of drilling individual holes. So it is an issue in the area of optimization. The article presents the results of simulation tests using the Finite Element Method in the form of the influence of selected hole drilling strategies on the temperature and thermal deformation of the plate. It has been clearly shown that increasing the number of simultaneously drilled holes (e.g. by using CNC multi-spindle drilling machines) shortens the time of their execution and may lead to a reduction in thermal deformation, if the adopted drilling strategy guarantees symmetry, i.e. a situation where the drilled holes remain symmetrical in relation to the axis of the plate. Deviation from the condition of symmetry of the drilled holes leads to a radical increase in thermal deformation of the plate. The conducted simulation tests also showed a certain scientific problem in the form of modeling the phenomenon of drilling a hole with (or not) taking into account the loss of material (eKill). The differences, especially in the temperatures, are so great that they can lead to wrong conclusions. However, it should be noted that modeling taking into account the phenomenon of disappearing material $(e K i l l)$ is extremely time-consuming and is ineffective without access to computers with high computing power.

The first, very simplified experiments showed that the plate will have to be flattened after drilling. Real thermal deformation depends very much on the fixing system. The paper did not investigate the effect of the fixing system.

\section{REFERENCES}

1. Collective work, (1974). Machine tools, Warsaw, Scientific and Technical Publishing WNT, (in Polish).

2. Kosmol J., (2021). Optimization of the drilling process in multi-hole objects in Modelling in Engineering 2020: Applied Mechanics, Series Advances in Intelligent Systems and Computing 1336, Springer International Publishing, doi: 10.1007/978-3-030-68455-6

3. Olszak W., (2008). Machining, Warsaw, Scientific and Technical Publishing WNT, (in Polish).

4. Pudlik W., (2012). Exchange and heat exchangers, Warsaw, Polish Scientific Publisher PWN, (in Polish).

5. Pająk, E., Wieczorowski, K., (1982). Basics of optimization of technological operations in the examples, Warsaw, Polish Scientific Publisher PWN, (in Polish).
6. Porada, G., (2020). Simulation tests by the Finite Element Method of temperature distribution and thermal deformation due to the heat generated during drilling, Gliwice, Thesis, Silesian University of Technology, (in Polish).

7. Wittbrod P., (2013). Optimization of the machining process with the use of genetic algorithms, www.ptzp.org.pl, (in Polish).

8.https://ansyshelp.ansys.com/account/secured?return url=/Views/Secured/search.html?q=commands\&lang $=$ en $\& \mathrm{qt}=\% 22$ default $\% 22 \&$ rows $=25 \& \mathrm{flt}=\% 22 \_$none_ $\% 22$

9. ANSYS 2019R2 https://www.ansys.com//media/ansys/en-gb/presentations/2019-innovationconference presentations $/ 2019$ - inn-confmechanical-update.pdf?la=en\&hash=

C85DB497D723C8BEB80AC1DA57BDBA7B30C7 $139 \mathrm{~A}$

Received: August 1, 2021 / Accepted: December 15, 2021 / Paper available online: December 20, 2021 (C) International Journal of Modern Manufacturing Technologies 